reactive protein were adjusted for in the analysis. Waist circumference (WC) and waist to hip ratio (WHR) were higher in the fourth quartile of serum ferritin than the third, also, in the third quartile compared to second quartile $(p<0.05) .31 \%$ and $52 \%$ of the women had excess abdominal obesity as indicated by WC and WHR respectively. The mean $(95 \% \mathrm{CI})$ serum transferrin receptor concentration $\{9.09 \mu \mathrm{g} / 1(8.77,9.44)\}$ was high indicating risk of iron deficiency. The mean $(95 \% \mathrm{CI})$ concentrations of lipids $\{\mathrm{TC}=4.78(4.64,4.93), \mathrm{HDL}-\mathrm{C}=1.45(1.39,1.52), \mathrm{LDL}-\mathrm{C}=1.65(1.53$, 1.78), $\mathrm{TG}=1.12(1.07,1.18) \mathrm{mmol} / \mathrm{l}\}$ were within recommended reference ranges.

Conclusions No significant association exists between iron status parameters and established CVD risk factors. But, excessive abdominal adiposity indicated by high WC and WHR contributes significantly to increased serum ferritin concentration in this population.

\section{P2-352 YOUTH HIV PREVALENCE AND SEXUAL BEHAVIOUR INDICATORS: EVIDENCE FROM NIGERIA}

doi:10.1136/jech.2011.142976k.84

\author{
${ }^{1,2} \mathrm{~A}$ Adeyemi, ${ }^{3} \mathrm{~A}$ Azeez, ${ }^{3} \mathrm{~K}$ Issa, ${ }^{4} 0$ Fakunle. ${ }^{1}$ MEASURE Evaluation, Abuja, Nigeria; \\ ${ }^{2}$ NACA, Abuja, Nigeria; ${ }^{3}$ Federal Ministry of Health, Abuja, Nigeria; ${ }^{4}$ University College \\ Hospital, Ibadan, Nigeria
}

Introduction Youths are particularly vulnerable to HIV/AIDS, sexual and reproductive health problems, which are major challenges to their health and development. There is a need to estimate HIV prevalence and understand predictors among them towards implementation of appropriate and evidence-based national interventions.

Methods A 2007 nationally representative household survey was analysed. It involved 4633 youths aged between 15 and 24 years. HIV prevalence and behavioural indicators were assessed. Logistic regression was used to model predictors of HIV infection.

Results Mean age was $19.1 \pm 2.8$ years; $50.9 \%$ male and $49.1 \%$ female. Youth HIV prevalence was $2.4 \%$ (national prevalence was $3.6 \%$ ); young women HIV prevalence was $2.9 \%$ and male counterpart $1.9 \%$. Sexual debut $<15$ years was $10.4 \% ; 7.8 \%$ exchanged sex for gifts; $28.3 \%$ had been away from home for $>1$ month; $18.4 \%$ had more than one sexual partner; $80.7 \%$ knew male condom protected against pregnancy; $75.6 \%$ knew male condom protected against HIV/AIDS. $81.9 \%$ had sexual intercourse in the last 12 months of which $45.8 \%$ used condom, and $10.5 \%$ were engaged in intergenerational sex with partners $\geq 10$ years. Risks for HIV infection were away from home for $>1$ month $\mathrm{AOR}=2.195 \%$ CI 1.3 to 2.7 ; being a female AOR $3.495 \%$ CI 2.8 to 6.7; sex without condom $\mathrm{AOR}=2.195 \% \mathrm{CI} 1.4$ to 5.6 while having at least secondary education was protective with AOR: $0.795 \%$ CI 0.3 to 0.9 .

Conclusion Youths accounted for $67 \%$ of HIV prevalence in Nigeria with young women disproportionately affected by HIV. Youths need appropriate and targeted behavioural interventions that involve partner reduction, consistent condom use and avoidance of intergenerational sex.

\section{P2-353 SOCIOECONOMIC TRENDS IN OBESITY IN EGYPT: CAN THE RISE IN PREVALENCE AND THE INCREASE IN INEQUALITIES BE PREVENTED?}

doi:10.1136/jech.2011.142976k.85

${ }^{1} \mathrm{~A}$ Aitsi-Selmi, ${ }^{2} \mathrm{M}$ Brown, ${ }^{2} \mathrm{~T}$ Marsh, ${ }^{1} \mathrm{M}$ G Marmot. ${ }^{1}$ University College London, London, UK; ${ }^{2}$ National Heart Forum, London, UK

Introduction Middle Eastern and North African countries have some of the highest obesity prevalence levels in the world (40\% among women in Egypt in 2008). The prevalence of obesity in low-andmiddle income countries has been rising in the last 2 decades and its socioeconomic distribution appears to be changing to the disadvantage of those with low socioeconomic status (SES) in many lowand-middle income countries.

Methods We first use five nationally representative survey waves (1992-2008) from Egypt to examine (1) prevalence trends; and (2) associations between SES and obesity using multivariate logistic regression and interaction tests. To help assess the policy implications, we are currently adapting the prediction model developed for the Foresight Tackling Obesities study in the UK to predict future obesity trends in Egypt.

Results Our regression analyses indicate that the rapid increase in obesity prevalence among low socioeconomic groups is the main factor driving the rise in overall prevalence. Adjusted coefficients of increase by education group were $7 \%$ (no education); 3\% (primary education); $2 \%$ (secondary education); $1 \%$ (higher education)-p-value for linear trend $<0.001$. Those most at risk appear to be those with low education and higher income $(p<0.05$ for an inhibitive interaction of education with income).

Conclusion Improving education levels appears to be an important policy approach to addressing both the prevalence rise and the socioeconomic inequalities in obesity in Egypt, particularly among lower socioeconomic groups experiencing rapid increases in income. Calculations from the prediction model will help quantify the impact of different educational policies on the obesity burden.

\section{P2-354 EFFECTIVENESS OF OPIOID SUBSTITUTION THERAPY AMONG PRISONERS WITH DRUG DEPENDENCE: A META ANALYSIS}

doi:10.1136/jech.2011.142976k.86

A Akinsemolu, ${ }^{*}$ S Ogston, L Irvine. University of Dundee, Dundee, UK

Background Illicit drug use is widespread in penal institutions worldwide. Despite this, the use of opioid substitution therapy (OST) in prisons is low. This study evaluates the effectiveness of OST in reducing the negative outcomes associated with opioid abuse among prisoners.

Methods Randomised controlled trials comparing OST with psychosocial therapy or no intervention for opioid dependent prisoners were included. Electronic searches were conducted in: MEDLINE, Embase, CINAHL, PsycINFO and the Cochrane Controlled Trials Register. Two reviewers independently identified relevant papers. Study quality was assessed using the Cochrane risk of bias tool. Pooled RRs were calculated using random effects models (with 95\% CI) for opioid use (measured by hair or urine analysis), and re-incarceration after release from prison.

Results Five studies with 820 participants met the inclusion criteria. Allocation concealment was adequate in one study and unclear in four. OST reduced opioid use (3 RCTs, RR 0.78 95\% CI 0.64 to 0.95 ) and re-incarceration (3 RCTs, RR $0.4195 \%$ CI 0.26 to 0.63 ). Two studies reported a statistically significant reduction in criminal activity and heroin use days, but the data were not pooled because of heterogeneity. Single studies reported statistically significant reductions in syringe sharing, mortality after release, cocaine use and retention in treatment.

Conclusion OST is in effective in reducing many negative outcomes associated with drug use among prisoners, giving far reaching benefits for prisoners, their families and society as a whole. OST should be widely available to prisoners with opioid dependence. 